Original Article

\title{
Evaluation of Open and Closed Kinetic Chain Exercises in Rehabilitation Following Anterior Cruciate Ligament Reconstruction
}

\author{
Mehmet UÇar, MD ${ }^{1 *}$, Irfan Koca, MD²), Mehmet Eroglu, MD ${ }^{3)}$, Selma Eroglu, MD ${ }^{4}$, \\ Umit Sarp, MD ${ }^{5}$, Hasan Onur Arik, MD ${ }^{6)}$, Alparslan Yetisgin ${ }^{2)}$ \\ 1) Department of Physical Medicine and Rehabilitation, Bozok University School of Medicine: Yozgat, \\ TR-66001, Turkey \\ 2) Department of Physical Medicine and Rehabilitation, Şanliurfa Education and Research Hospital, \\ Turkey \\ 3) Department of Orthopaedics and Traumatology, Mardin State Hospital, Turkey \\ 4) Department of Physical Medicine and Rehabilitation, Mardin State Hospital, Turkey \\ 5) Department of Physical Medicine and Rehabilitation, Yozgat State Hospital, Turkey \\ 6) Department of Orthopaedics and Traumatology, Yozgat State Hospital, Turkey
}

\begin{abstract}
Purpose] To compare outcomes of anterior cruciate ligament (ACL) reconstruction after open kinetic chain (OKC) exercises and closed kinetic chain (CKC) exercises. [Subjects and Methods] The subjects comprised 11 female and 47 male patients who are randomly divided into two groups: which performed a CKC exercise program Group I and Group II which performed an OKC exercise program. Pain intensity was evaluated using visual analogue scale (VAS). Knee flexion was evaluated using a universal goniometer, and thigh circumference measurements were taken with a tape measure at baseline and at 3 months and 6 months after the treatment. Lysholm scores were used to assess knee function. [Results] There were no significant differences between the two groups at baseline. Within each group, VAS values and knee flexion were improved after the surgery. These improvements were significantly higher in the $\mathrm{CKC}$ group than in the OKC group. There were increases in thigh circumference difference at the 3 and 6 month assessments post-surgery. A greater improvement in the Lysholm score was observed in the $\mathrm{CKC}$ group at 6 months. [Conclusion] The CKC exercise program was more effective than OKC in improving the knee functions of patients with ACL reconstruction.

Key words: Anterior cruciate ligament reconstruction, Closed kinetic chain exercises, Open kinetic chain exercises
\end{abstract}

(This article was submitted Apr. 23, 2014, and was accepted May 29, 2014)

\section{INTRODUCTION}

Anterior cruciate ligament (ACL) tears are prevalent musculoskeletal injuries among physically active individuals and are most frequently seen in the population aged 15-25 years ${ }^{1,2)}$. ACL reconstruction is the standard operative method used to prevent the progress of unwanted musculoskeletal complications. There are many graft options, fixation techniques, and postoperative rehabilitation programs for the treatment of ACL tears. Arthroscopically assisted ACL reconstruction with the use of autograft or allograft tissue is generally preferred in surgery ${ }^{3}$. Rehabilitation methods constitute an important part of the treatment aiming to reduce pain and joint effusion, improve the range of motion (ROM) of the knee, and increase quadriceps

*Corresponding author. Mehmet Uçar (E-mail: drmehmetucar@msn.com)

(C2014 The Society of Physical Therapy Science. Published by IPEC Inc. This is an open-access article distributed under the terms of the Creative Commons Attribution Non-Commercial No Derivatives (by-ncnd) License $<$ http://creativecommons.org/licenses/by-nc-nd/3.0/> . strength following ACL reconstruction ${ }^{4)}$. However, there is no consensus on the ideal timeframe and the type of exercises that should be prescribed ${ }^{5)}$.

Data in the literature suggests both open and closed kinetic chain $(\mathrm{CKC})$ exercise treatment after ACL reconstructive surgery. Closed kinetic chain exercises have gained popularity over open kinetic chain (OKC) exercises because many clinicians believe that $\mathrm{CKC}$ exercises are more reliable and functional ${ }^{6}$. Supporters of CKC exercise also note that it is more effective than OKC exercise at restoring quadriceps femoris muscle strength after ACL reconstruction surgery. CKC exercises have also been reported to exert more strain on the reconstructed ACL and are less harmful to the patellofemoral joint ${ }^{7}$.

The aim of this study was to evaluate OKC and CKC exercises, and to report the early and mid-term results of patients performing $\mathrm{CKC}$ and $\mathrm{OKC}$ exercise programs after $A C L$ reconstructive.

\section{SUBJECTS AND METHODS}

The subjects were 58 patients with unilateral ACL tears 
who underwent arthroscopically assisted ACL reconstruction with an autograft of hamstring tendons.

Approval for the study was granted by the Local Ethics Committee of Bozok universty and informed consent was received from all the study participants. From the initial 66 consecutive patients admitted to the outpatient clinic, 58 were randomly allocated into 2 groups by the sealed envelope method. Following a detailed physical examination, a standard evaluation form was completed for each patient. Demographic information including sex, age, weight, height, body mass index, occupation, education level, pain intensity and affected side were recorded. Preoperative and post-treatment tests were applied for 4 variables of pain intensity, thigh circumference difference, knee flexion, and Lysholm score. The presence of ACL tear was confirmed by physical examination and magnetic resonance imaging at the initial visit. All subjects were required to be older than 17 years of age and underwent arthroscopically assisted ACL reconstruction surgery.

Subjects with a history of other lower extremity pathology and trauma, previous surgery of the lower extremity, and inflammatory disorders or other rheumatic diseases, neurological or psychiatric disorders, other problems related to either knee, or with a history of drug therapy other than analgesics or physiotherapy treatment within the last 3 months were excluded. Patients were randomly allocated to Group I (CKC) or Group II (OKC).

Arthroscopically assisted ACL reconstruction was performed with a double bundle autologous hamstring graft using the rigidfix system (The DePuy Mitek RIGIDFIX Cross Pin System) for all the patients with ACL tear by the same surgeons (ME, HOA). In Group I, 19 patients had also meniscus degeneration - tear (16 medial, 3 lateral). In Group II, 17 patients also had meniscus degeneration - tear (15 medial and 2 lateral). All meniscial lesions were partially removed.

A Jones bandage, elevation and cold pack were applied immediately after the operation. The following rehabilitation programme was followed by both groups. The patients were encouraged to stand and bear weight using crutches 24 hours postoperatively. The knee was fully extended by over press. Between Days 3-7, ankle pump exercises were conducted (4 sets of 20 repetitions), knee isometric quadriceps flexion exercises ( 3 sets of 50 repetitions) and straight leg elevation of $0-50^{\circ}$ ( 3 sets of 20 repetitions). Passive knee flexion-extension between $0-90^{\circ}$ with a continuous passive movement device and walking with crutches with full weight-bearing on the operated side was started between days 7-15. The CKC and OKC exercises shown in Table 1 were performed ( 3 sets of 20 repetitions). When knee flexion reached $110^{\circ}$ at $15-30$ days, the patients were allowed to walk quickly, run on a smooth surface and ascend and descend stairs.

Outcome measurements were taken preoperatively, then at 3 months and 6 months after surgery in both groups. Subjectively perceived pain intensity was assessed using visual analog scale (VAS) $(0-100 \mathrm{~mm})$, active knee flexion was evaluated with a universal goniometer, and thigh circumference difference was calculated from measurements made
Table 1. Exercises protocols of both groups

\begin{tabular}{ll}
\hline $\begin{array}{l}\text { Group I (CKC) } \\
\mathrm{N}=30\end{array}$ & $\begin{array}{l}\text { Group II (OKC) } \\
\mathrm{n}=28\end{array}$ \\
\hline Squatting lunges exercise & Isometric quadriceps exercise \\
Standing weight shift exercise & Flexor-extensor bench \\
Wall sits exercise & Isotonic quadriceps exercise \\
One-legged quad dips exercise & Long leg press on-off exercise \\
Lateral step-ups exercise & Knee flexion-extension \\
& stretching exercise \\
\hline
\end{tabular}

CKC: closed kinetic chain; OKC: open kinetic chain

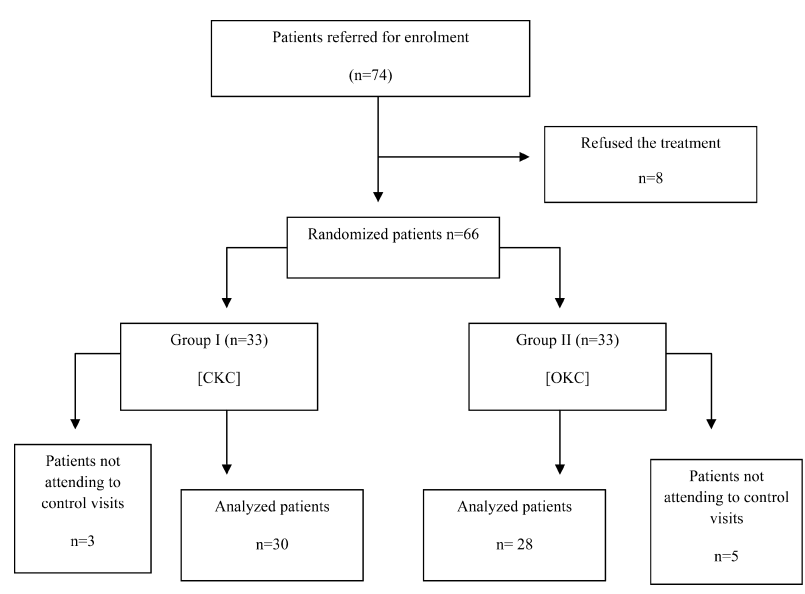

Fig. 1. Flow diagram of the study process, indicating patient selection

CKC: closed kinetic chain; OKC: open kinetic chain

with a tape measure. The thigh circumference difference was measured $15 \mathrm{~cm}$ above the upper rim of the patella ${ }^{8)}$ and represents the circumference measurement difference between the operated and normal thighs. Lysholm scores were used to assess the knee function, as this is an accepted scale of patient functionality demonstrating patient satisfaction in daily activities of mobility such as walking, ascending and descending stairs and squatting ${ }^{9)}$

Non-nominal baseline and outcome data were tested for the normal distribution using the Kolmogorov-Smirnov test. Results were expressed as frequencies or mean $\pm \mathrm{SD}$. The baseline differences between the groups were then analyzed using Student's t-test and one-way ANOVA with a post-hoc test (Dunnets test) for repeated measures analysis of variance. The differences of categorical variables between the groups were tested by $\chi^{2}$ analysis.

\section{RESULTS}

Initially 74 consecutive patients, aged $17-39$ years, who were admitted to the Physical Medicine and Rehabilitation outpatient clinic following ACL reconstruction were enrolled in the study. Sixty-six patients who met the eligibility criteria of the study were randomly allocated two groups according to the sequence of allocation (Fig. 1).

The CKC group comprised 30 patients and the $\mathrm{OKC}$ 
Table 2. Baseline characteristics of the patients

\begin{tabular}{lcc}
\hline & $\begin{array}{c}\text { Group I } \\
(\mathrm{CKC})\end{array}$ & $\begin{array}{c}\text { Group II } \\
(\mathrm{OKC}) \\
\mathrm{n}=30\end{array}$ \\
\hline Age (years) & $27.4 \pm 10.5$ & $28.1 \pm 11.9$ \\
Sex (male/female) & $24 / 6$ & $23 / 5$ \\
BMI $\left(\mathrm{kg} / \mathrm{m}^{2}\right)$ & $26.9 \pm 1.8$ & $25.6 \pm 1.9$ \\
Duration of diagnosis (months) & $3.9 \pm 1.4$ & $4.1 \pm 1.6$ \\
Affected side (right/left) & $14 / 16$ & $10 / 18$ \\
Education (years) & $9.6 \pm 4.2$ & $8.5 \pm 3.2$ \\
\hline CKC: closed kinetic chain; OKC: open kinetic chain; BMI: body \\
mass index & & \\
Values are presented as mean $\pm \mathrm{SD}$ &
\end{tabular}

group comprised 28 patients. Baseline characteristics of the patients are given in Table 2. There were no significant differences between the two groups regarding baseline characteristics (all $\mathrm{p}$ values $>0.05$ ).

The baseline values of VAS, thigh circumference difference, Lysholm score and knee flexion were similar between the two groups (Table 3). However, significant decreases in VAS scores at 3 and 6 months post-surgery were found in both groups and the VAS score decreased more in the CKC group than in the OKC group. Knee flexion showed significant increases at 3 and 6 months in both groups, and the values were greater in the CKC group than in the OKC group. Significant improvements in the Lysholm score were found at 6 months post-surgery in both groups (both $p>0.05$ ), and the improvement was greater in the $\mathrm{CKC}$ group than in the OKC group. There were increases in thigh circumference difference at the 3 and 6 month assessments and the increases were significant in both groups at 3 months postsurgery (Table 3).

\section{DISCUSSION}

The results of this study show that the VAS pain scores of in both groups decreased after treatment and the decrease in these scores was much greater in the CKC group than in the OKC group. There were increases in thigh circumference differences at 3 and 6 months post-surgery, and the increases were significant in both groups at 3 months post-surgery. The knee flexion values and Lysholm scores of both groups increased after surgery and the increases in the knee flexion and Lysholm scores were larger in the CKC group than in the OKC group.

For successful ACL reconstruction, a special rehabilitation programme is essential for decrease of pain and inflammation, for regaining functional range of motion for undertaking daily living and sporting activities, and for regaining previous quality of life. However, there is no consensus on the post-surgery rehabilitation regimen In rehabilitation following ACL reconstruction, it has been proposed that the graft and knee should be protected via longer immobilization, and that weight-bearing should be avoided to prevent instability ${ }^{10)}$. However, Shelbourne et al., reported that weight-bearing and accelerated rehabilitation programmes including full extension of the knee on the first day had fa-
Table 3. The comparison of the groups' clinical findings

\begin{tabular}{lcc}
\hline & $\begin{array}{c}\text { Group I } \\
(\mathrm{CKC}) \\
\mathrm{n}=30\end{array}$ & $\begin{array}{c}\text { Group II } \\
(\mathrm{OKC}) \\
\mathrm{n}=28\end{array}$ \\
\hline VAS 100 $(\mathrm{mm})$ & & \\
Baseline & $72.3 \pm 11.4$ & $65.6 \pm 12.6$ \\
3 months & $41.4 \pm 12.9 *$ & $48.6 \pm 11.4 *$ \\
6 months & $22.1 \pm 10.5 *$ & $27.2 \pm 9.9 *$ \\
Thigh circumference difference (cm) & \\
Baseline & $1.1 \pm 0.8$ & $1.2 \pm 0.9$ \\
3 months & $3.2 \pm 1.8 *$ & $4.1 \pm 2.1 *$ \\
6 months & $1.3 \pm 1.5$ & $1.6 \pm 1.4$ \\
Knee flexion $\left({ }^{\circ}\right)$ & & \\
Baseline & $40.8 \pm 17.6$ & $45.4 \pm 16.7$ \\
3 months & $125.6 \pm 27.1 *$ & $110.9 \pm 24.1 *$ \\
6 months & $135.1 \pm 16.1 *$ & $128.5 \pm 18.1 *$ \\
Lysholm scores & & \\
Baseline & $66.3 \pm 12.4$ & $64.2 \pm 10.1$ \\
3 months & $80.8 \pm 19.1$ & $78.5 \pm 14.5$ \\
6 months & $94.1 \pm 8.5 *$ & $84.3 \pm 9.1 *$ \\
\hline
\end{tabular}

CKC: closed kinetic chain; OKC: open kinetic chain; VAS: Visual Analog Scale

${ }^{*}$ The intragroup analysis revealed significant improvements after treatment when compared with baseline $(\mathrm{p}<0.05)$

Values are presented as mean $\pm \mathrm{SD}$

vorable effects on knee stability ${ }^{11)}$.

In the present study, full extension and weight-bearing were achieved on the first postoperative day. In the early postoperative assessments at both 3 and 6 months, no instabilities were seen. We consider this is one of the positive effects of early rehabilitation and is consistent with the results of some previous studies ${ }^{12,13)}$

Yack et al. compared OKC and CKC exercises in ACL rehabilitation and reported that there was more laxity in the OKC group ${ }^{14)}$. However, Perry et al. found no knee laxity differences between $\mathrm{OKC}$ and $\mathrm{CKC}$ groups ${ }^{15)}$. In the present study, no laxity was determined in either group.

Postoperative knee edema and swelling develop immediately, and in the following days, muscle weakness and atrophy begin in the quadriceps muscle, indicating the need for exercise therapy to maintain the muscle mass. The most significant thigh circumference difference was seen at 3 months in the present study. This difference was lower in the CKC group, which can be considered to be the result of $\mathrm{OKC}$ exercises increasing the hamstring and quadriceps muscle forces simultaneously and earlier than CKC exercises. The thigh circumference differences were equal in the OKC and CKC groups at the end of the 6th month.

The essential reasons for performing OKC and CKC exercises are to avoid loss of muscle strength, preserve knee $\mathrm{ROM}$, and to maintain knee functionality and proprioception. In the present study, although both $\mathrm{CKC}$ and $\mathrm{OKC}$ had positive effects on knee flexion, the $\mathrm{CKC}$ exercises were more effective at inhibiting muscle atrophy of the knee flexors and extensors at 3 and 6 months.

These results are similar to those of Barrett ${ }^{16)}$ and show 
that $\mathrm{CKC}$ exercises performed while weight-bearing are more effective at muscle strengthening and increasing joint range of motion. CKC exercises are considered to be better for restoring normal knee function and weight-bearing ${ }^{17)}$.

Bynum et al. compared ROM and Lysholm scores between an $\mathrm{OKC}$ group and a CKC group and found significantly higher values in the $\mathrm{CKC}$ group ${ }^{6}$. In the present study, there were statistically significant differences between the groups in the Lysholm and ROM scores of the patients, and the values of the CKC group were higher at the end of the 6th month. This was contrary to the findings of a study by Hooper et al., who reported that there were no differences between OKC and CKC groups at the 4th week $^{18)}$. As the postoperative measurements were taken at later times in the present study, it is possible that performing the exercises for longer periods may have a more positive effect on the results.

In a study by Morrissey et al., VAS pain scores of OKC and $\mathrm{CKC}$ groups were compared and no differences were found between the groups ${ }^{13}$. That finding was in contrast to the results of the present study, which show VAS scores were lower in the CKC group at both 3 and 6 months postsurgery. This can be explained by CKC exercises being conducted with weight-bearing, which improves the early mobilization and functionality of the patients. In addition, Hooper et al., reported 4-week values, a shorter observation period than that of the present study; thus, CKC exercises performed regularly for a longer period affects the results ${ }^{18)}$

In conclusion, the results of this study demonstrate that in the rehabilitation of $\mathrm{ACL}$ reconstruction, $\mathrm{CKC}$ exercises are more effective than OKC exercises, at providing mobilization and enabling a quicker return to daily and sporting activities.

\section{REFERENCES}

1) Griffin LY, Agel J, Albohm MJ, et al.: Noncontact anterior cruciate ligament injuries: risk factors and prevention strategies. J Am Acad Orthop Surg, 2000, 8: 141-150. [Medline]

2) Meisterling S, Schoderbek R, Andrews JR: Anterior cruciate ligament reconstruction. Oper Tech Sports Med, 2009, 17: 3-10. [CrossRef]

3) Smith MA, Smith WT, Kosko P: Anterior cruciate ligament tears: reconstruction and rehabilitation. Orthop Nurs, 2014, 33: 14-24, quiz 25-26.
[Medline] [CrossRef]

4) Palmieri-Smith RM, Thomas AC, Wojtys EM: Maximizing quadriceps strength after ACL reconstruction. Clin Sports Med, 2008, 27: 405-424, vii-ix. [Medline] [CrossRef]

5) Atkinson HDE, Laver JM, Sharp E: Physiotherapy and rehabilitation following soft-tissue surgery of the knee. Orthop Trauma, 2010, 24: 129-138. [CrossRef]

6) Bynum EB, Barrack RL, Alexander AH: Open versus closed chain kinetic exercises after anterior cruciate ligament reconstruction. A prospective randomized study. Am J Sports Med, 1995, 23: 401-406. [Medline] [CrossRef]

7) Fitzgerald GK: Open versus closed kinetic chain exercise: issues in rehabilitation after anterior cruciate ligament reconstructive surgery. Phys Ther, 1997, 77: 1747-1754. [Medline]

8) Jakobsen TL, Christensen M, Christensen SS, et al.: Reliability of knee joint range of motion and circumference measurements after total knee arthroplasty: does tester experience matter? Physiother Res Int, 2010, 15 : 126-134. [Medline] [CrossRef]

9) Cho SH, Bae CH, Gak HB: Effects of closed kinetic chain exercises on proprioception and functional scores of the knee after anterior cruciate ligament reconstruction. J Phys Ther Sci, 2013, 25: 1239-1241. [Medline] [CrossRef]

10) Risberg MA, Lewek M, Snyder-Mackler L: A systematic review of evidence for anterior cruciate ligament rehabilitation: how much and what type? Phys Ther Sport, 2004, 5: 125-145. [CrossRef]

11) Shelbourne KD, Nitz P: Accelerated rehabilitation after anterior cruciate ligament reconstruction. Am J Sports Med, 1990, 18: 292-299. [Medline] [CrossRef]

12) Morrissey MC, Hudson ZL, Drechsler WI, et al.: Correlates of knee laxity change in early rehabilitation after anterior cruciate ligament reconstruction. Int J Sports Med, 2000, 21: 529-535. [Medline] [CrossRef]

13) Morrissey MC, Hudson ZL, Drechsler WI, et al.: Effects of open versus closed kinetic chain training on knee laxity in the early period after anterior cruciate ligament reconstruction. Knee Surg Sports Traumatol Arthrosc, 2000, 8: 343-348. [Medline] [CrossRef]

14) Yack HJ, Collins CE, Whieldon TJ: Comparison of closed and open kinetic chain exercise in the anterior cruciate ligament-deficient knee. Am J Sports Med, 1993, 21: 49-54. [Medline] [CrossRef]

15) Perry MC, Morrissey MC, King JB, et al.: Effects of closed versus open kinetic chain knee extensor resistance training on knee laxity and leg function in patients during the 8- to 14 -week post-operative period after anterior cruciate ligament reconstruction. Knee Surg Sports Traumatol Arthrosc, 2005, 13: 357-369. [Medline] [CrossRef]

16) Barrett DS: Proprioception and function after anterior cruciate reconstruction. J Bone Joint Surg Br, 1991, 73: 833-837. [Medline]

17) Grodski M, Marks R: Exercises following anterior cruciate ligament reconstructive surgery: biomechanical considerations and efficacy of current approaches. Res Sports Med, 2008, 16: 75-96. [Medline] [CrossRef]

18) Hooper DM, Morrissey MC, Drechsler W, et al.: Open and closed kinetic chain exercises in the early period after anterior cruciate ligament reconstruction. Improvements in level walking, stair ascent, and stair descent. Am J Sports Med, 2001, 29: 167-174. [Medline] 